\title{
Algorithmic Improvement Of Dynamic Hand Gesture Recognition Based On HMM Algorithm
}

\author{
Xue Xue, Zhuojia Li, Chuyu Hong \\ Key Lab of Media Audio \& Video of Ministry of Education \\ Communication University of China, Beijing 100024, China \\ xuexue@cuc.edu.cn, lizhuojia0301@126.com,mazdycyh.student@sina.com
}

\begin{abstract}
Compared with other methods of dynamic gesture recognition, dynamic hand gesture recognition based on Hidden Markov Model (HMM) is more widely used. This method aims at analyzing hand signals. In traditional HMM method, any gestures would be calculated with the largest probability as the final recognition result according to Forward - Backward algorithm [1]. In this paper, We study and propose improved algorithm by using some methods like graphics normalizing, probability range limit, number of points limit, coding species limit, etc. to enhance the HMM method for improving the recognition rate and reducing the false detection rate. Experimental results show that the gesture trajectory recognition rate of such method is about $94 \%$, which is higher than the traditional rate of $80 \%$. Furthermore, the excluding error recognition rate, which was not dealt in the past, has reached $100 \%$. Besides, our algorithm can be widely used because of its simple method of equipment, high precision and better robustness.
\end{abstract}

Keywords- Dynamic gesture recognition; HMM algorithm;

threshold method; Forward-Backward algorithm; recognition rate

\section{INTRODUCTION}

Dynamic gesture recognition is widely used recently, which aims at problems of gesture recognition rate and we propose a solution to improve it. As dynamic gesture recognition is part of the core, the main methods in the field of identification technology are various, such as Dynamic gesture recognition based on histogram, Dynamic gesture recognition based on Dynamic Time Warping (DTW), Dynamic gesture recognition based on Hidden Markov Mode (HMM) ,etc. Among them, Dynamic gesture recognition based on histogram misses the time characteristics of the sequence [2], Dynamic gesture recognition based on Dynamic Time Warping (DTW) is very time-consuming with a large amount of calculation with higher dependence on the endpoint detection[3], and Dynamic gesture recognition based on Hidden Markov Mode (HMM) has advantages in expansibility, time scale invariance, automatic segmentation and classification [4]. Considering the analysis above, dynamic gesture recognition based on Hidden Markov Mode (HMM) model is adopted.

Dynamic hand gesture recognition using the traditional HMM method can only be used to identify effective gestures, and it cannot exclude invalid or undefined gestures. If we must identify a hand gesture which is not defined in the template library, then, as a result, the undefined gesture will be mistaken as an effective gesture. Therefore, in order to eliminate invalid gestures, improving the recognition rate and reducing the detection error rate became the must. For this, we should use various methods to improve the HMM algorithm.

In this paper, we put forward the improvement of the traditional solution of the HMM algorithm, and eventually improve the gesture recognition rate and reduce invalid gesture error detection rate at the same time. In section II, we briefly review the background knowledge of Dynamic gesture recognition and HMM algorithm. In section III, we introduce the threshold method and analyze the results, and then put forward our own solution. Finally, we compare the results of the experiments and come to the conclusions.

\section{BACKGROUND KNOWLEDGE}

\section{A. Dynamic gesture recognition based on Kinect}

The process of the dynamic gesture recognition is: firstly, use Microsoft Kinect camera to acquire the gesture's depth map, and change the depth map into three-dimensional point cloud; then get the palms point coordinate data to be encoded according to the depth information of filter and use the mainstream method-HMM to train the coding into Hidden Markov sample library; at last, use the HMM to do real-time dynamic gesture recognition [5]. Fig. 1 is the schematic diagram of Dynamic gesture recognition .

\section{B. Description of HMM algorithm}

Hidden Markova Model (HMM) is a kind of statistical properties of probability model which is used to describe random process [6]. And it is also a double stochastic process. It consists of two parts: the Markova chain and general stochastic process. The Markova chain is used to describe the transfer of states, and expressed in transition probability. General stochastic process is used to describe the states and the relationship between the observed sequences, and expressed in observation probability.

In the experiment, we collect respectively in dynamic gesture recognition as "up", "down", "left", "right", "stop", "open", "end", the seven defined styles of gesture 
trajectories. Samples of each of the trajectory coordinates, which uses Baum - Welch algorithm, are trained to HMM model respectively. Then we need to test dynamic gesture recognition with HMM method. When making sample identification test with such method, we should always use Forward-Backward arithmetic [7] to identify the test samples. We test sample generation into every gesture model to calculate Backward probability, then compare the calculation of probability, and finally regard the biggest probability as the recognition result. For negative samples (undefined gestures), the trajectory according to the probability will be mistakenly identified as effective gestures because the traditional HMM method does not handle on it. When we deal with real-time sample test with HMM method [8], palms trajectory coordinates obtained from the program in real time will be stored in an array, and then we extract the characteristic value using the same method beforehand. Finally we substitute it into the HMM model, calculate the probability, and identify the results. Fig. 2 is the HMM training schematic diagram.

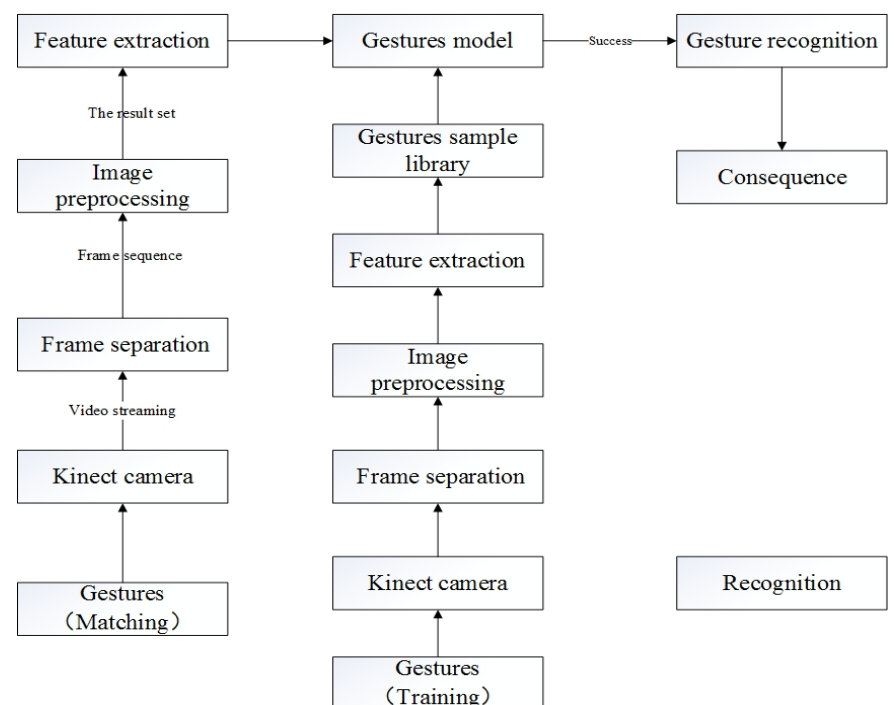

Figure1. Schematic diagram of Dynamic gesture recognition

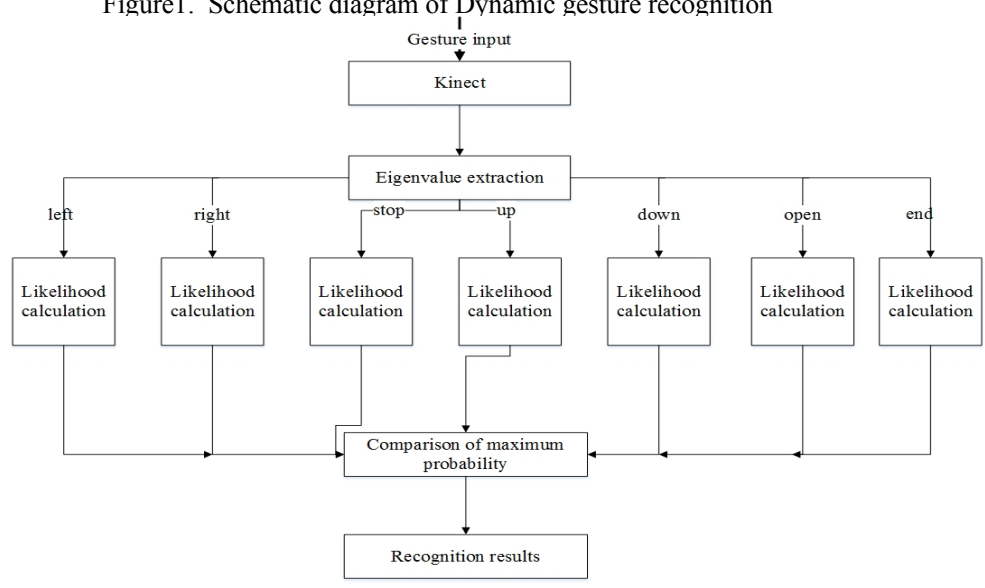

Figure2. HMM training schematic diagram

\section{STUDY AND IMPROVING}

\section{A. Eliminate invalid gestures with Threshold method}

We use threshold model method proposed by South Korean Hyeon-Kyu Lee and Jin H. Kim [9] to distinguish the defined and undefined gestures. Threshold model is a fully connected model. According to previous scholars studying the threshold model for years, threshold model can solve problems of how to distinguish the defined gestures and undefined gestures better.

Specific threshold model can be represented as:

$$
\lambda_{\mathrm{T}}=\left\{\pi_{\mathrm{T}}, \mathrm{A}_{\mathrm{T}}, \mathrm{B}_{\mathrm{T}}\right\}
$$

In (1), the specific threshold can be calculated as:

$$
\begin{gathered}
{\left[\begin{array}{ccccc}
a_{11} & \frac{1-a_{11}}{N-1} & \frac{1-a_{11}}{N-1} & \ldots & \frac{1-a_{11}}{N-1} \\
\frac{1-a_{22}}{N-1} & a_{22} & \frac{1-a_{22}}{N-1} & \ldots & \frac{1-a_{22}}{N-1} \\
\frac{1-a_{33}}{N-1} & \frac{1-a_{33}}{N-1} & a_{33} & \ldots & \frac{1-a_{33}}{N-1} \\
\cdots & \ldots & \ldots & \ldots & \ldots \\
\frac{1-a_{N N}}{N-1} & \frac{1-a_{N N}}{N-1} & \frac{1-a_{N N}}{N-1} & \ldots & a_{N N}
\end{array}\right] \text { (2) }} \\
\operatorname{In}(2), N=N_{1}+N_{2}+\ldots+N_{k}, N_{i}(\mathrm{i}=1,2 \ldots \mathrm{k}) \text { is the }
\end{gathered}
$$
condition number of gestures' model, $k$ is the species number of all hand gestures, $a_{i i}$ is the transition probability of all states in the model.

$$
\begin{array}{r}
\mathrm{B}_{1} \\
\mathrm{~B}_{2} \\
\mathrm{~B}_{T}=\mathrm{B}_{3} \\
\cdots \\
\mathrm{B}_{k}
\end{array}
$$

In (3), $\mathrm{B}_{T}$ is the observation probability matrix.

$$
\pi_{T}=\left[\begin{array}{lllll}
\frac{1}{k} \pi_{1} & \frac{1}{k} \pi_{2} & \frac{1}{k} \pi_{3} & \ldots & \frac{1}{k} \pi_{k}
\end{array}\right]
$$

In (4), $\pi_{T}$ is the initial probability distribution of gestures.

We follow the method of threshold model for processing the parameters, but in the end we find that this method has a great influence on our own system. Although the invalid gestures can be excluded, but at the same time it also excludes a lot of effective gestures. Even we have changed some of the parameters, the effect is still poor. The reason is that the threshold value obtained by using the model is too high, even obviously higher than the maximum effective gesture probability. Therefore, we must submit algorithm improvement in view of our own system. 


\section{B. Put forward our own methods for improvement}

We have improved traditional HMM method, resulting in increasing recognition rate and ineffective gesture's false detection rate. The main improvements of the algorithm are: normalizing the sample trajectory into the same size to reduce the influence of trajectory shape size on recognition rate; setting the range of sample path probability by using statistical regularity to eliminate invalid gestures; using the number of track points range to eliminate invalid gestures; using coding species to reduce false detection rate, etc. The details are below:

1) Normalize the sample trajectory into the same size

In order to eliminate the influence of the size and shape of the trajectory of the gesture, we normalize the image to reduce the influence of trajectory shape size on recognition rate.

2) Set the range of sample path probability using statistical regularity

a) Add up the probability of each gesture using Forward-Backward algorithm respectively, and then set up probability group. According to statistical regularity, general scope of every gesture probability is obtained.

b) Modify Forward-Backward algorithm, if the identified gesture of probability is beyond the scope of regulation gesture of probability, the gesture is considered invalid.

c) After setting up effective gestures of probability range, input the corresponding effective gestures and invalid gestures to test whether the scope is right.

3) Use the number of track points range

Using the former method can eliminate most invalid gestures, but for some particular gestures, this method is difficult to exclude invalid gestures. For example, the trajectory "C" would be identified as gesture trajectory "O". The reason is that such gestures and the definition of effective gestures are too similar, thus probability will fall within the scope of the effective gestures set, and the gestures will be identified as effective ones. Therefore, we use the number of track points range to eliminate invalid gestures. The steps are:

a) Add up the general scope of each valid gestures point number respectively according to the statistical regularity.

b) Modify Forward-Backward algorithm, input the number of signal points. When gestures point number is beyond the scope of effective gestures point number, the gestures will be considered invalid.

c) After setting up effective gestures' point number range, input the corresponding effective gestures and invalid gestures to detect the number of points.

4) Use coding species to reduce false detection rate

In order to further reduce the false detection rate, on the basis of the third method, we also use coding species to reduce false detection rate. For some invalid gestures, if they meet the probability of the scope of the second method and the number of points of the third way at the same time, the invalid gestures may not be excluded. To this end, we use coding species to further processing. The steps are:

a) Add up the coding species of effective gestures according to the statistical regularity.

b) Modify Forward-Backward algorithm, input gestures coding species. When gestures coding species is beyond the scope of effective gestures coding species, gestures will be considered invalid.

c) After setting up effective gestures coding species range, then input the corresponding effective gestures and the particular invalid gestures to detect the signal coding range of species.

5) Use the first code of gesture to decrease the missing gesture

After using the methods mentioned above, most of the missing gestures can be removed by the suitable threshold. But the gesture " 3 " sometimes was recognized as the gesture "E". For this reason, we designed a special method using the range of code for this gesture. The steps are:

a) Add up the gesture " $E$ " range of first code method

b) Modify Forward-Backward Histogram, take the value of the first code method, if the value was not in the correct gesture code range, the histogram would recognize this gesture as missing gesture.

c) After having adjusted the histogram, process the gesture " 3 " and gesture " $E$ " to check if the code range was suitable.

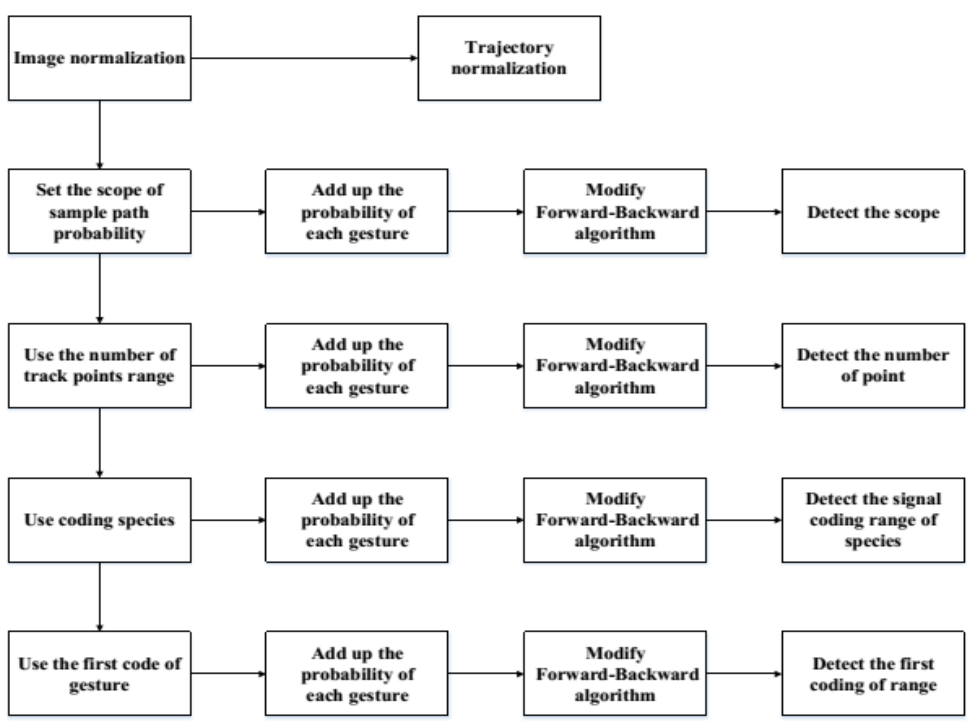

Figure 3. Flow chart of our new improved algorithm 


\section{EXPERIMENT DETAILS}

In the experiments, we do the sample test and real-time test [10] respectively, and compare their identification results with traditional testing. We select 50 samples in our sample test to complete "up", "down", "left", "right", "stop", "open", "end", the seven kinds of gesture recognition and negative samples (i.e. invalid gestures) of gesture recognition. Finally we identify the sample correctly and calculate their recognition rate respectively. Similarly, in real time test, we select the three people to input 50 samples, a total of $3 \times 50$ samples for testing. In the end, we calculate the final recognition rate and compare it with the results of sample test. Table I shows the results using threshold method without our algorithm improvement, while Table II shows the results improved by our algorithm.

TABLE I. Results Using Threshold Method

\begin{tabular}{|c|c|c|c|c|c|c|c|}
\hline & Traditional & \multicolumn{3}{|c|}{ Sample test } & \multicolumn{3}{|c|}{ Real time testing } \\
\hline gesture & $\begin{array}{c}\text { recognition } \\
\text { rate }\end{array}$ & total number & $\begin{array}{c}\text { proper } \\
\text { identification }\end{array}$ & $\begin{array}{c}\text { reeognition } \\
\text { rate }\end{array}$ & total number & $\begin{array}{c}\text { proper } \\
\text { iddentification }\end{array}$ & $\begin{array}{c}\text { recognition } \\
\text { rate }\end{array}$ \\
\hline right & - & 50 & 39 & $78.00 \%$ & $3 \times 50$ & 128 & $85.33 \%$ \\
\hline left & - & 50 & 42 & $84.00 \%$ & $3 \times 50$ & 135 & $90.00 \%$ \\
\hline up & - & 50 & 50 & $100 \%$ & $3 \times 50$ & 138 & $92,00 \%$ \\
\hline down & - & 50 & 38 & $76.00 \%$ & $3 \times 50$ & 135 & $90.00 \%$ \\
\hline stop & $80 \%$ & 50 & 8 & $16.00 \%$ & $3 \times 50$ & 20 & $13,33 \%$ \\
\hline open & $70 \%$ & 50 & 15 & $30.00 \%$ & $3 \times 50$ & 11 & $7.33 \%$ \\
\hline end & $75 \%$ & 50 & 5 & $10.00 \%$ & $3 \times 50$ & 6 & $4.00 \%$ \\
\hline negative samples & - & 50 & 50 & $100 \%$ & $3 \times 50$ & 150 & $100 \%$ \\
\hline
\end{tabular}

TABLE II. RESULTS USING OUR IMPROVED ALGORITHM

\begin{tabular}{|c|c|c|c|c|c|c|c|}
\hline Category & \multicolumn{3}{|c|}{ Sample test } & \multicolumn{3}{c|}{ Real time testing } \\
Type & $\begin{array}{c}\text { Traditional } \\
\text { test }\end{array}$ & \multicolumn{3}{|c|}{} \\
\hline gesture & $\begin{array}{c}\text { recognition } \\
\text { rate }\end{array}$ & total number & $\begin{array}{c}\text { proper } \\
\text { identification }\end{array}$ & $\begin{array}{c}\text { recognition } \\
\text { rate }\end{array}$ & total number & $\begin{array}{c}\text { proper } \\
\text { identification }\end{array}$ & $\begin{array}{c}\text { recognition } \\
\text { rate }\end{array}$ \\
\hline right & - & $\mathbf{5 0}$ & 49 & $98.00 \%$ & $3 \times 50$ & 149 & $99.33 \%$ \\
\hline left & - & $\mathbf{5 0}$ & 49 & $98.00 \%$ & $3 \times 50$ & 149 & $99.33 \%$ \\
\hline up & - & $\mathbf{5 0}$ & $\mathbf{5 0}$ & $100 \%$ & $3 \times 50$ & 150 & $100.00 \%$ \\
\hline down & - & $\mathbf{5 0}$ & 49 & $98.00 \%$ & $3 \times 50$ & 149 & $99.33 \%$ \\
\hline stop & $80 \%$ & $\mathbf{5 0}$ & 47 & $94.00 \%$ & $3 \times 50$ & 147 & $98.00 \%$ \\
\hline open & $70 \%$ & $\mathbf{5 0}$ & 46 & $92.00 \%$ & $3 \times 50$ & 147 & $98.00 \%$ \\
\hline end & $75 \%$ & 50 & 49 & $98.00 \%$ & $3 \times 50$ & 142 & $94.67 \%$ \\
\hline negative samples & - & $\mathbf{5 0}$ & $\mathbf{5 0}$ & $100 \%$ & $3 \times 50$ & 150 & $100 \%$ \\
\hline
\end{tabular}

\section{CONCLUSION AND DisCUSSION}

After comparing Table II with Table I, we can see that the gesture trajectory recognition rate of our methods is about $94 \%$, higher than the traditional rate $80 \%$, and excluding error recognition rate, which was not dealt in the past, has reached $100 \%$. Besides, our algorithm can be widely used because of its simple method of equipment, high precision and better robustness.

Through our five kinds of methods above mentioned, the recognition rate of gesture recognition can be improved effectively, and ineffective gesture recognition rate can be further reduced.

\section{ACKNOWLEDGMENT}

This work is supported by the National Nature Science Foundation of China (61201236, 61371191), and Engineering Planning Fund of Communication University of China (HG1008, 3132013XNG1350, 3132013XNG1338), Nation Science and Technology Support Program (2012BAH39F01-05).

\section{REFERENCES}

[1] Wikipedia.Forward-backward-algorithm, the free encyclopedia. http://en.wikipedia.org/wiki/Forwardbackward_algorithm.[Online;last modified on 30-Octo.

[2] Qing Yang. "Hand Gesture Recognition". Dalian University of Technology,2010.12.

[3] Hong P, Turk M, Huang T S. "Gesture modeling and recognition using finite machines". In: Proceedings of the 4th International Conference on Face and Gesture Recognition. 2000. 410 415.

[4] M. Vafadar,A. Behrad, "Human Hand Gesture Recognition Using Motion Orientation Histogram for Interaction of Handicapped Persons with Computer",the 2008 International Conference on Image and Signal Processing, Cherbourg(France), LNCS 5099, $2008: 378-385$.

[5] S. Mitra and T. Acharya, Gesture recognition: a survey, IEEE Transactions on Systems, Man, and Cybernetics (SMC) C Part C: Applications andReviews 37 (3), 2007, pp.311-324.

[6] Andrew D. Wilson, Aaron F. Bobick, "Hidden Markov Models for Modeling and Recognizing Gesture under Varia- tion". International Journal of Pattern Recognition and Arti- ficial Intelligence (IJPRAI), Volume 15, Number 1, February 2001,pp.123-160.

[7] Wikipedia.Baum-Welch-algorithm,the free encyclopedia. http://en.wikipedia.org/wiki/Baum-Welch_algorithm.[Online;last modified on 1-April -2011].

[8] R. I. A. Davis and B. C. Lovell, "Comparing and Evaluating HMM Ensemble Training Algorithms Using Train and Test and Condition Number Criteria." To Appear in the Journal of Pattern Analysis and Applications, 2003.

[9] Hyeon-Kyu Lee and Jin H.Kim. An HMM-Based Threshold Model Approach for Gesture Recognition. IEEE TRANSACTIONS ON PATTERN ANALYSIS AND MACHINE INTELLIGENCE, VOL.21,NO.10,OCTOBER 1999,PP961-973.ber -2011].

[10] F. S. Chen, C. M. Fu and C. L. Huang, Hand gesture recognition using a real-time tracking method and hidden Markov models, Image and Vision Computing 21, 2003, pp.745-758. 